\title{
Simulated hypogravity impairs the angiogenic response of endothelium by up-regulating apoptotic signals
}

\author{
Lucia Morbidelli $^{\mathrm{a}, *, 1}$, Monica Monici ${ }^{\mathrm{b}, 1}$, Nicola Marziliano ${ }^{\mathrm{c}}$, Augusto Cogoli ${ }^{\mathrm{d}}$, \\ Franco Fusi ${ }^{\mathrm{e}}$, Johannes Waltenberger ${ }^{\mathrm{f}}$, Marina Ziche ${ }^{\mathrm{a}}$ \\ a Section of Pharmacology, Department of Molecular Biology and C.R.I.S.M.A., University of Siena, Siena, Italy \\ ${ }^{\mathrm{b}}$ CEO-Centro di Eccellenza Optronica, Firenze, Italy \\ ${ }^{\mathrm{c}}$ Laboratorio di Genetica Molecolare, IRCCS Policlinico San Matteo, Pavia, Italy \\ ${ }^{\mathrm{d}}$ Space Biology, ETH Zurich, Switzerland \\ e Department of Clinical Physiopathology, University of Florence, Florence, Italy \\ ${ }^{\mathrm{f}}$ Department of Interventional Cardiology, University of Maastricht, The Netherlands
}

Received 20 June 2005

Available online 1 July 2005

\begin{abstract}
Health hazards in astronauts are represented by cardiovascular problems and impaired bone healing. These disturbances are characterized by a common event, the loss of function by vascular endothelium, leading to impaired angiogenesis. We investigated whether the exposure of cultured endothelial cells to hypogravity condition could affect their behaviour in terms of functional activity, biochemical responses, morphology, and gene expression. Simulated hypogravity conditions for $72 \mathrm{~h}$ produced a reduction of cell number. Genomic analysis of endothelial cells exposed to hypogravity revealed that proapoptotic signals increased, while antiapoptotic and proliferation/survival genes were down-regulated by modelled low gravity. Activation of apoptosis was accompanied by morphological changes with mitochondrial disassembly and organelles/cytoplasmic NAD $(\mathrm{P}) \mathrm{H}$ redistribution, as evidenced by autofluorescence analysis. In this condition cells were not able to respond to angiogenic stimuli in terms of migration and proliferation. Our study documents functional, morphological, and transcription alterations in vascular endothelium exposed to simulated low gravity conditions, thus providing insights on the occurrence of vascular tissue dysregulation in crewmen during prolonged space flights. Moreover, the alteration of vascular endothelium can intervene as a concause in other systemic effects, like bone remodelling, observed in weightlessness.
\end{abstract}

(C) 2005 Elsevier Inc. All rights reserved.

Keywords: Endothelial cell; Hypogravity; Gene expression; Autofluorescence; Apoptosis; Angiogenesis; Migration; Proliferation

Angiogenesis, a process which leads to formation of new vessels, has a relevant physiological role during development and in the adult life [1,2]. Endothelial cells are the true player of the angiogenesis process. Several cardiovascular pathologies are caused by endothelial dysfunction (myocardial ischemia, atherosclerosis), thus growth factors and strategies able to restore the integrity

\footnotetext{
${ }^{*}$ Corresponding author. Fax: +39 0577234343.

E-mail address: morbidelli@unisi.it (L. Morbidelli).

${ }^{1}$ These authors equally contributed to the work.
}

of the endothelium and to promote angiogenesis can be viewed as innovative therapeutic approaches $[3,4]$.

Indications that mechanical stimulation of cells, and endothelium in particular, is able to change some of their functions at biochemical and molecular level are reported in the literature [5]. In spite of the evidence that cells are sensitive to mechanical stimuli, the molecular mechanisms by which individual cells recognize and respond to external forces are not well understood. Our interest on hypogravity originates from the recognition that several disturbances associated to endothelium- 
dependent functionality (as myocardial injury, altered wound healing, osteoporosis, and endocrine organ dysfunction) have been found in crewman on prolonged space flights and in animal models [6,7]. Endothelial cells seem very sensitive to the absence or reduction of gravity. Literature data describe a reduction in cell replication [8], even if some reports show opposite results [9]. Whether hypogravity may affect cell functions (i.e., gene expression) and apoptosis signals in endothelium remains to be elucidated.

In order to evaluate how conditions of simulated hypogravity could affect endothelial cell behaviour, we designed studies to evaluate gene expression, metabolism, and cell functions. Expression profiling of selected genes was evaluated by quantitative RT-PCR, while multicolour imaging autofluorescence microscopy was used to define the morphological and biochemical alterations, indexes of the metabolic status of the cells and the occurrence of apoptosis [10,11]. Moreover vascular endothelial cells exposed to hypogravity were assessed for their responses to angiogenic growth factors in terms of chemotaxis and proliferation. All together, our results demonstrate that the exposure to simulated hypogravity conditions impairs endothelial cell survival, promoting gene up- and down-regulation, biochemical and morphological features leading to apoptosis and inability to respond to angiogenic factors.

\section{Materials and methods}

Cell lines and culture conditions. Porcine aortic endothelial cells (PAEC) overexpressing vascular endothelial growth factor receptor-2 (VEGFR-2) [12] were maintained with Ham's F-12 medium supplemented with $10 \%$ fetal calf serum (FCS) and $500 \mu \mathrm{g} / \mathrm{ml} \mathrm{G} 418$ sulfate antibiotic. PAEC were split weekly 1:3.

Simulation of hypogravity conditions. PAEC were seeded in T25 flasks in a subconfluent condition. After adhesion, flasks were completely filled (to avoid shear stress) with medium containing 10\% FCS and exposed to hypogravity conditions, simulated by a random positioning machine (RPM) (Fokker Space, Leiden, The Netherlands) for $72 \mathrm{~h}$. The angular velocity of the rotation was $60 \%$. Temperature was maintained at $37^{\circ} \mathrm{C}$. Control samples $(1 \mathrm{~g})$ were treated as those kept in the RPM and placed close to the RPM. In the RPM probes are fixed as close as possible to the center of two frames rotating one inside the other, driven by separate motors [13]. The rotation of each frame is random and autonomous under computer control. A software drives the motors in a smooth, continuous (i.e., joltless) movement. The angular velocity of the rotation can be selected between $30^{\circ}$ and $360^{\circ} \%$. An electronic box installed on the inner frame powers the experiment (temperature control, sensors, and illumination) and provides the connection for on-line video recording.

Immediately after hypogravity exposure, cells were lysed for gene expression or detached and used for functional/morphological assays (in normal gravity).

Quantitative RT-PCR for gene expression. Cells exposed for $72 \mathrm{~h}$ to hypogravity $\left(1 \times 10^{6}\right.$ cells/T25) were resuspended in $2 \times$ lysis buffer (Applied Biosystems). Total RNA extraction was made by using the ABI PRISM 6100 platform according to the manufacturer's instructions. RNA samples were then resuspended in a final volume of $100 \mu \mathrm{l}$ of elution buffer for spectrophotometric yield quantitation. The aver- age RNA was $80 \pm 5 \mathrm{ng} / \mu \mathrm{l}$. Total RNA (100 ng) was reverse transcribed using a high-capacity cDNA Archive Kit (Applied Biosystems), random hexamer primers, and the following thermal profile: $25^{\circ} \mathrm{C}$ for $10 \mathrm{~min}, 42^{\circ} \mathrm{C}$ for $1 \mathrm{~h}$, and $95^{\circ} \mathrm{C}$ for $5 \mathrm{~min}$. For all of the further analyses, the cDNA was diluted 1:20 in water.

The screened genes for apoptosis and proliferation are listed in Table 1. For each of which we made use of Assay on Demand FAMMGB-labeled probes. We used the GAPDH reference gene (VICMGB-labeled probe; PN 4326317E), which was always co-amplified in the same wells with the target genes in order to normalize the different amounts of total RNA in the different wells, and their RT reaction efficiencies. RT-PCR was performed using the automated ABI Prism 7900 HT Sequence Detector System (Applied Biosystems). The cDNAs were also tested for gDNA contamination, whose overall level was no higher than $0.005 \%$.

Differentially expressed genes were quantitated by using the threshold cycle $\left(C_{\mathrm{t}}\right)$ and the comparative method $\left(2^{-\Delta \Delta C_{\mathrm{t}}}\right)$ [14]. The control cell lines (normal gravity) were used as calibrator sample.

Autofluorescence studies. To provide information on morphological and functional state of living cells, autofluorescence analysis was performed. Cells contain molecules, engaged both in structural and functional processes, which become fluorescent under a suitable excitation by UV-Vis irradiation. The emission of these endogenous fluorophores is called autofluorescence to distinguish it from the fluorescence obtained by adding exogenous markers. The most important endogenous fluorophores are proteins containing aromatic amino acids, the reduced form of pyridine nucleotides, flavins, and lipopigments $[15,16]$.

Cells previously exposed to hypogravity and control condition were detached and plated on glass coverslips at the density of $5 \times 10^{4}$ cells/ $\mathrm{cm}^{2}$. Endothelial cell autofluorescence was analysed by an inverted epifluorescence microscope (Nikon Diaphot) equipped with an oilimmersion CF-UV fluor 100× objective (NA 1.3), under $365 \mathrm{~nm}$ excitation from a filtered $(10 \mathrm{~nm}$ bandwidth interference filter, 365FS10-25, Andover) high-pressure mercury lamp (HBO $100 \mathrm{~W}$, Osram). The fluorescence signal, transmitted through a dichroic mirror at $400 \mathrm{~nm}$ (DM400, Nikon), was detected, according to the position of a mobile mirror, by a diode-array spectrophotometer (based on ARC polichromator equipped with a Hires III cooled digital CCD camera (DTA, Italy) and a backilluminated SITe $330 \times 1100$ pixels sensor), through an optical fiber bundle (1 $\mathrm{mm}$ diameter), or by a Hires III digital CCD camera (DTA, Italy) equipped with a Kodak KAF261E, $512 \times 512$ pixels, detector. Therefore, the system allowed sequential measurements of spectra and images on the same cell.

In front of the CCD detector, a motorized filter wheel, which can hold up to eight different interference filters, allowed a multispectral sequential acquisition under software control by the host computer. The choice of the filter series was made on the basis of the main

Table 1

Genes studied in this work

\begin{tabular}{ll}
\hline Gene name & Assay on Demand \\
\hline BAX & Hs00180269_m1 \\
FAS & Hs00169544_m1 \\
FAS-L & Hs00181225_m1 \\
p53 & Hs00364137_m1 \\
PCNA & Hs00427214_g1 \\
BCL2 & Hs00236808_s1 \\
RANK & Hs00187189_m1 \\
RANK-L & Hs00187387_m1 \\
\hline
\end{tabular}

The genes screened in the present study and their related Assay on Demand code are reported; enter the code name in the My Science page (www.appliedbiosystems.com) for further information such as exon/exon boundaries and splice variants. 
spectral bands determined by preliminary analysis of the autofluorescence spectra of the different cell populations.

Proliferation studies. Cell proliferation was quantified by total cell number as reported [17]. Briefly, after adherence of sparse cells (previously exposed to hypogravity) the supernatant was replaced with $1 \%$ serum medium containing increasing concentrations of serum, the angiogenic factors fibroblast growth factor-2 (FGF-2) [18] and VEGF165 splice variant [19], and incubated at $1 \mathrm{~g}$ for further $48 \mathrm{~h}$. Cells were fixed with methanol and stained with Diff-Quik. Cell numbers were obtained by counting in seven random fields at a magnification of 100 with the aid of an ocular grid. Data are reported as number of cells counted/well.

Migration assay. Cell migration was assessed in 48-well microchemotaxis chambers (NeuroProbe, Biomap, Milan, Italy) on a polycarbonate filter, $8 \mu \mathrm{m}$ pore size, coated either with type I collagen and fibronectin as reported [17]. A cell suspension containing 12,500 cells previously exposed to hypogravity, was added to the upper chamber of each well and angiogenic stimuli to the lower one. After $4 \mathrm{~h}$ of incubation at $1 \mathrm{~g}$, cells which did not migrate were removed and the filter was stained with Diff Quik (Biomap, Milan, Italy). Migrated cells were counted in 10 random fields/well at a magnification of 400. Data are reported as number of migrated cells counted/well.

Statistical analysis. For functional assays, multiple comparisons were performed by one-way analysis of variance (ANOVA) and individual differences tested by Fisher's test after the demonstration of significant inter-group differences by ANOVA. A $p$ value lower than 0.05 was considered statistically significant. For gene expression data analysis, the two groups were compared by means of one-way ANOVA, with $p$ values of less than 0.001 being considered statistically significant. The $\sigma$ values of every target gene in each gene panel between the groups (which were always less than $0.05 \%$ ) support the reliability of the generated data. In order to assess the reproducibility of the method, we calculated the coefficient of variation for each gene, which was never more than $0.67 \%$.

Reagents. Cell culture reagents and materials were from Sigma Chemical, St. Louis, MO, USA. FCS was from Hyclone, Logan, UT, USA. Human recombinant FGF-2 and VEGF165 were from Calbiochem-Novabiochem Int., San Diego, CA, USA. Diff-Quik was from Mertz-Dade AG, Dudingen, Switzerland.

\section{Results}

\section{Simulated hypogravity alters gene expression pattern in endothelial cells}

Simulated hypogravity condition (for $72 \mathrm{~h}$ ) produced impressive changes in cell morphology, resulting in a marked elongated shape compared to control cells. Simulated hypogravity also reduced the number of cells $(20 \%)$ recovered after detachment (approximately $3.5 \times 10^{6}$ cells/T25 flask in control condition vs $0.7 \times 10^{6}$ cells/T25 flask in hypogravity condition).

To characterize the molecular mechanisms for reduced cell number and altered morphology, control and hypogravity-exposed cells were studied for gene expression profiling of apoptosis and proliferation/survival signals.

Fig. 1 shows the expression trends in hypogravity and control cells. The expression of apoptotic genes was statistically higher in the hypogravity exposed cells, while anti-apoptotic genes were up-regulated in control cells.

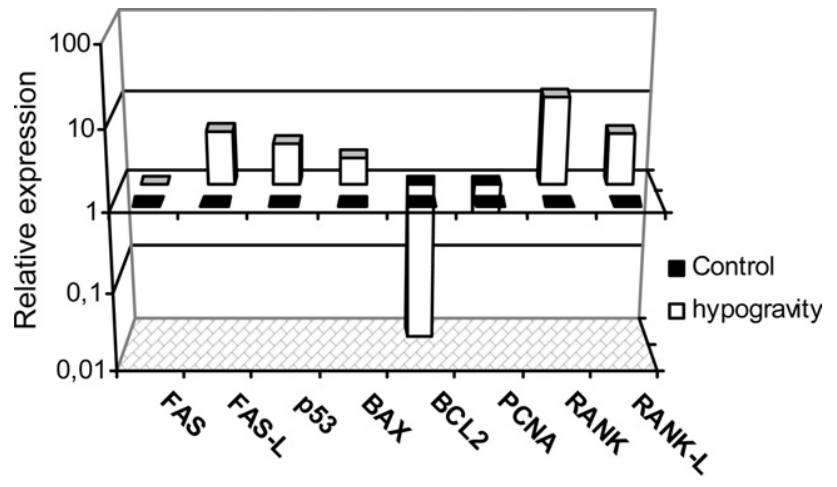

Fig. 1. Gene expression in endothelial cells exposed to hypogravity condition. PAEC cells exposed for $72 \mathrm{~h}$ to hypogravity and $1 g$ conditions were lysed and total RNA was extracted. Differentially expressed genes for proliferation and apoptosis signalling cascades were quantitated by using the comparative $C_{\mathrm{t}}$ method. Data are reported as time-fold over- or down-expression of the pro-apoptotic and anti-apoptotic genes in hypogravity cells, taking control cells as reference (fold increase $=1$ ).

In particular, the expression of p53 (TP53), a key player in the apoptotic process due to DNA damage, was higher (3.2 times) in hypogravity exposed cells. Active p53 transactivates pro-apoptotic genes, including BAX (2.1 times higher in hypogravity) in order to promote full apoptosis. The typical death receptor FAS - along with the TNF R-was expressed to similar extent in control and hypogravity cells, while the FAS-L was higher (4.5 times) in hypogravity-exposed cells. Interestingly, in hypogravity exposed cells the expression of the anti apoptotic gene BCL-2 was only $1 \%$ of that found in control cells (i.e., BCL-2 expression was 101 times higher in control than in hypogravity cultures).

Moreover, the PCNA transcript, a marker of cell mitosis, was also found to be decreased in the absence of gravity (Fig. 1). RANK (receptor activator of NK-kB) and RANK-L, biochemical markers of bone remodelling and inflammatory reactions [20], were overexpressed in hypogravity condition (Fig. 1).

\section{In hypogravity exposed cells multicolour autofluorescence analysis reveals morphological and biochemical changes associated with apoptosis}

In order to evaluate the biochemical changes associated with the gene expression profiling seen above, autofluorescence analysis on single living cell has been performed by excitation at $365 \mathrm{~nm}$, particularly suitable for $\operatorname{NAD}(\mathrm{P}) \mathrm{H}$, but able to excite flavins too.

In Fig. 2, the autofluorescence pattern of endothelial cells exposed to simulated hypogravity (B and C) is shown and compared with the control one (A). In the latter the blue component, mainly due to the reduced form of the nicotinic coenzymes, has a discrete cytoplasmic location (bound to the organelles) and masks the green component, mostly due to flavins, arising from 


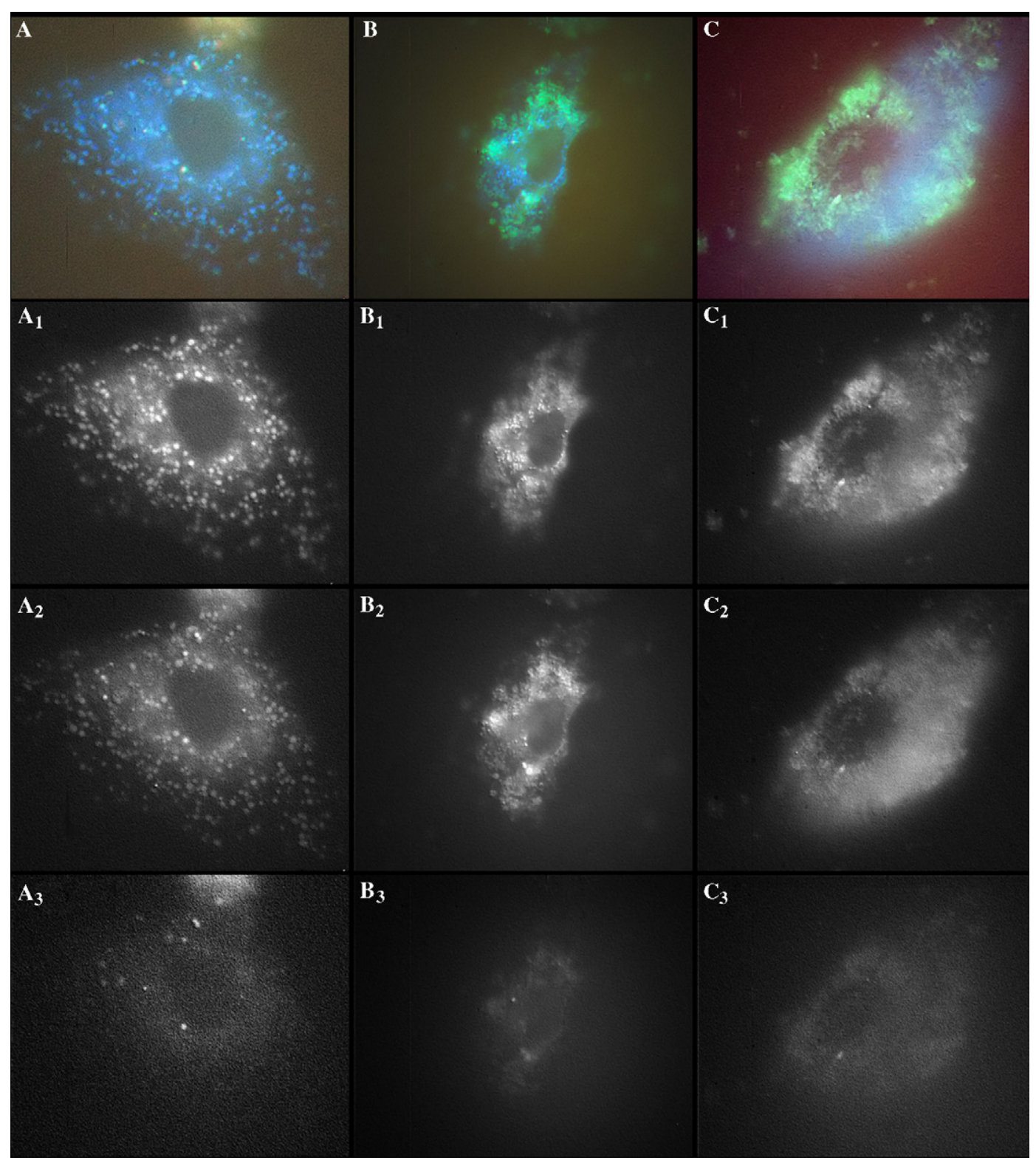

Fig. 2. Autofluorescence pattern of endothelial cells cultured at $1 g(\mathrm{~A})$ and in simulated hypogravity conditions (B,C). Fluorescence images were directly digitized in the CCD controller with a 14 bit dynamics and transferred to the storage computer on a digital interface. The size of the field detected by the $100 \times$ objective was about $69 \times 69 \mu \mathrm{m}$ (spatial calibration of $0.13 \mu \mathrm{m} \mathrm{pixel}^{-1}$ ), as determined by imaging $6 \mu \mathrm{m}$ fluorescent microspheres (Molecular Probes). For each sample, three $40 \mathrm{~nm}$ wide (full-width at half maximum) spectral bands, peaked at about 450, 550, and $650 \mathrm{~nm}$ (450 FS 40-25, 550 FS 40-25, and 650 FS 40-25 respectively, Andover, Salem, NH) were used in order to sequentially acquire three fluorescence images in blue, green, and red, with integration times of about $5 \mathrm{~s}$. Monochrome images were then combined in a single multicolour image using the RGB technique [33]. The multicolour images were obtained by the Image Combine Channels algorithm of Corel PHOTO-PAINT v 6.0 software (Corel Corporation, Ottawa, Canada), after the identification of the three greyscale images (acquired at 650, 550, and $450 \mathrm{~nm}$, respectively) with the RGB components. In control cells $(1 g)$, autofluorescence emission arises from cytoplasmic organelles and the distribution of the blue $(\mathrm{NAD}(\mathrm{P}) \mathrm{H})$ and green (flavines) components is very similar (see $A_{1}, A_{2}$, and $A_{3}$ ). In hypogravity-cultured cells, blue and green components have different distribution and a disruption of the organelles occurs (see $\mathrm{B}_{1}, \mathrm{~B}_{2}, \mathrm{~B}_{3}$ and $\mathrm{C}_{1}, \mathrm{C}_{2}, \mathrm{C}_{3}$ ). (For interpretation of the references to colour in this figure legend, the reader is referred to the web version of this paper.)

the same sites $\left(A_{1}, A_{2}\right.$, and $\left.A_{3}\right)$. On the contrary, in the RPM-exposed cells the blue and green components have different intracellular distribution, with a less evident location inside the organelles. In more than $70 \%$ of the cells, blue and green fluorescent blurs appear and a progressive dissolution of intracellular structures can be observed. Moreover, most of hypogravity exposed cells show cell shrinkage as well as convolution of nuclear outline, typical features of apoptosis (Figs. 2B and C).

The comparative ratio among the major pools of intracellular coenzymes (reduced/oxidized forms of pyridinic and flavinic coenzymes, respectively, bound 

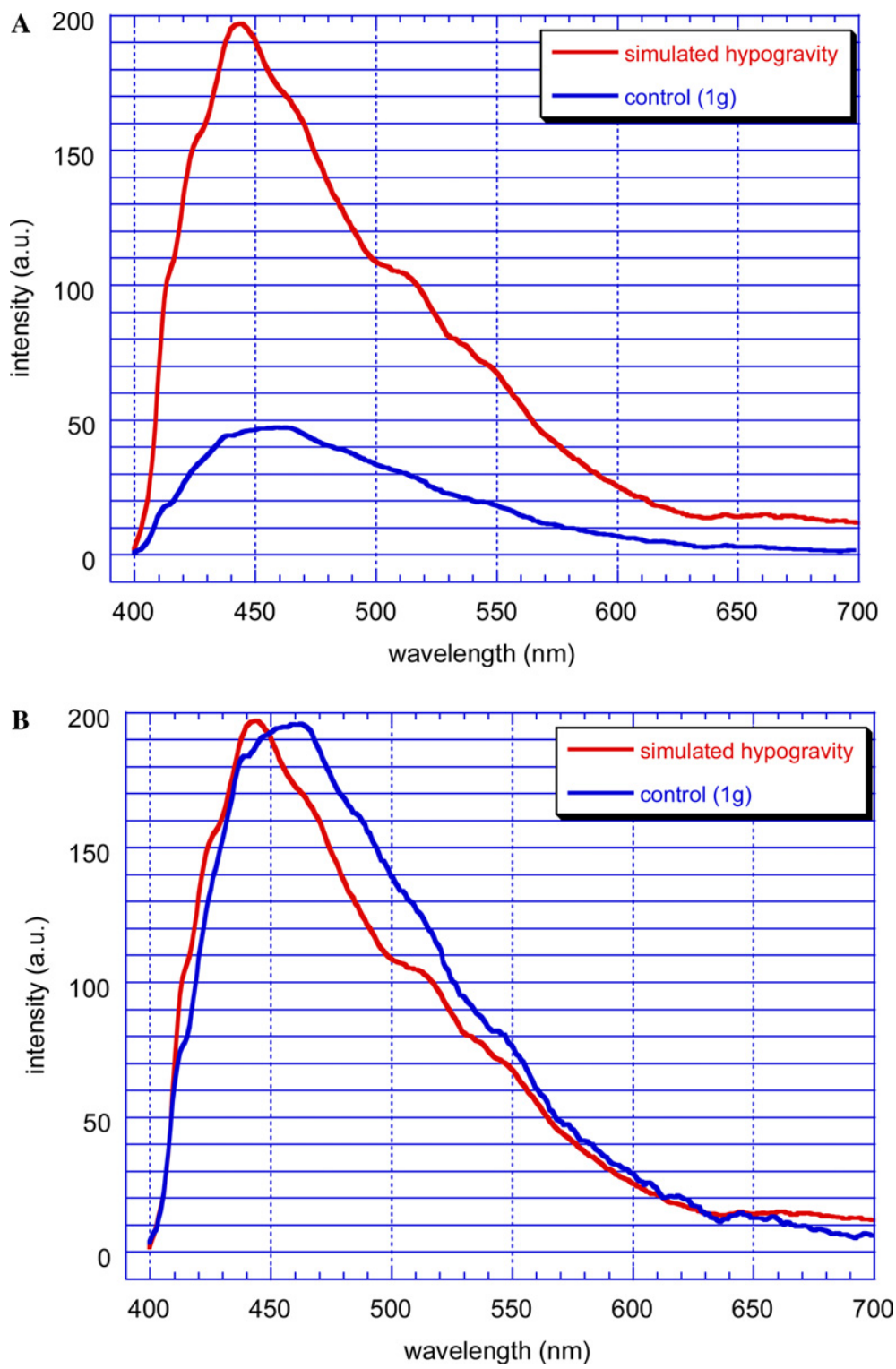

Fig. 3. (A) Autofluorescence spectra of endothelial cells cultured at $1 g$ (blue) and in simulated hypogravity conditions (red). The emission intensity in hypogravity-exposed cells is higher (about threefold) than in the control and the main peak, corresponding to the NAD(P)H emission, is blue-shifted. Fluorescence spectra were recorded through a band-pass filter (GG400, Schott) to remove UV excitation, and collected over a spot of about $10 \mu \mathrm{m}$ in diameter, as estimated by using $6 \mu \mathrm{m}$ fluorescent microspheres (F-14808, Molecular Probes, Leiden, The Netherlands). Fluorescence spectra, recorded with a 2-4 s integration time, were corrected for the optical system spectral response and smoothed by a gaussian convolution algorithm. After correction, spectra were normalized to their respective peaks as $100 \%$ for comparison (B). (For interpretation of the references to colour in this figure legend, the reader is referred to the web version of this paper.)

to the proteins $(\mathrm{NAD}(\mathrm{P}) \mathrm{Hb})$ and free $(\mathrm{NAD}(\mathrm{P}) \mathrm{Hf})$ forms of the pyridinic ones) can be better examined by autofluorescence microspectroscopy. Thus, the spectrum of treated cells was compared with the control one both for the emission intensity (Fig. 3A) and spectral shape after normalization (Fig. 3B). The autofluorescence emission of the hypogravity-exposed cells was higher (about threefold) than that of the control. Moreover, the main peak, corresponding to the NAD $(\mathrm{P}) \mathrm{H}$ emission, appeared blue-shifted in comparison with the control.
In Table 2, the ratios between the autofluorescence intensity values at $450 / 480,450 / 525$, and $480 / 525 \mathrm{~nm}$, from spectra of cells kept on the RPM and controls are reported. The wavelengths examined $(450,480$, and $525 \mathrm{~nm}$ ) correspond to the peaks of NADHb, NADHf, and flavins, respectively. Therefore, analysis of these ratios provides insights on the balances among the different coenzymatic pools. In hypogravity-exposed cells the ratio $450 / 525 \mathrm{~nm}$ remained unchanged, even if the total emission greatly increased, in comparison with controls. This means that both $\mathrm{NAD}(\mathrm{P}) \mathrm{H}$ and oxidized 
Table 2

Ratios of autofluorescence intensity values at 450, 480, and $525 \mathrm{~nm}$ in hypogravity exposed cells and controls

\begin{tabular}{lll}
\hline $\begin{array}{l}\text { Ratio description } \\
(\text { wavelengths) }(\mathrm{nm})\end{array}$ & $\begin{array}{l}\text { Simulated hypogravity } \\
(\text { value } \pm \sigma)\end{array}$ & $\begin{array}{l}\text { Control }(1 g) \\
\text { (value } \pm \sigma)\end{array}$ \\
\hline $450 / 480$ & $1.27 \pm 0.10$ & $1.14 \pm 0.02$ \\
$450 / 525$ & $1.85 \pm 0.33$ & $1.85 \pm 0.22$ \\
$480 / 525$ & $1.46 \pm 0.20$ & $1.63 \pm 0.19$ \\
\hline
\end{tabular}

The considered wavelengths $(450,480$, and $525 \mathrm{~nm})$ correspond to the peaks of NADHb, NADHf, and flavins, respectively. Mean values calculated on 10 cells have been reported. The ratio $450 / 525 \mathrm{~nm}$ has the same value, while the ratios 450/480 and 480/525 $\mathrm{nm}$ increased and decreased, respectively, in hypogravity exposed cells compared to the control. This demonstrates the decrease of intracellular NAD $(\mathrm{P}) \mathrm{Hf}$ as well as the increase of NAD $(\mathrm{P}) \mathrm{Hb}$.

flavins increased and accumulated in the cells. The ratios $450 / 480$ and $480 / 525 \mathrm{~nm}$ increased and decreased, respectively, in comparison with the control, thus pointing out depletion of the NAD(P)Hf pool and enhancement of the $\mathrm{NAD}(\mathrm{P}) \mathrm{Hb}$ one, according to the blue shift of the spectrum.

Simulated hypogravity impairs endothelial cell functions in response to angiogenic factors

Based on the above results we ascertained whether cell impairment could affect endothelium to mount an angiogenic response. Following enzymatic detachment, an equal number of cells from hypogravity and control cultures was exposed to angiogenic stimuli and then monitored for changes in proliferation and migration in normal gravity.

Cell proliferation in response to increasing concentration of serum, VEGF165 or FGF-2 was affected by hypogravity (Fig. 4). Control cells proliferated in response to increasing concentrations of serum (1-10\%), but hypogravity reduced by $50 \%$ their growth when counted after $48 \mathrm{~h}$ of incubation, suggesting that cells did not progress through the cell cycle (Fig. 4A). VEGF165, acting as pro-angiogenic stimulus [19], produced concentration related $(2-20 \mathrm{ng} / \mathrm{ml}$ range $)$ cell growth in control cells, while it failed to promote growth in cells exposed to weightlessness (Fig. 4B). Not only cells were insensitive to their physiological agonist (VEGF), but they were not able to respond to other mitogens as FGF-2. Indeed, FGF-2 nearly doubled the cell number at 10 and $20 \mathrm{ng} / \mathrm{ml}$ (maximal at $10 \mathrm{ng} / \mathrm{ml}$ ) in control conditions, while it failed to affect cell growth in cells previously grown in the absence of gravity (Fig. $4 \mathrm{C})$.

These data suggest that hypogravity exposure irreversibly changed cell biochemical and signalling machinery, thus not allowing cell survival and recovery once exposed to proangiogenic stimuli. Moreover, other cell functions than cell growth were affected by the absence of gravity. The exposure to gradients of increasing
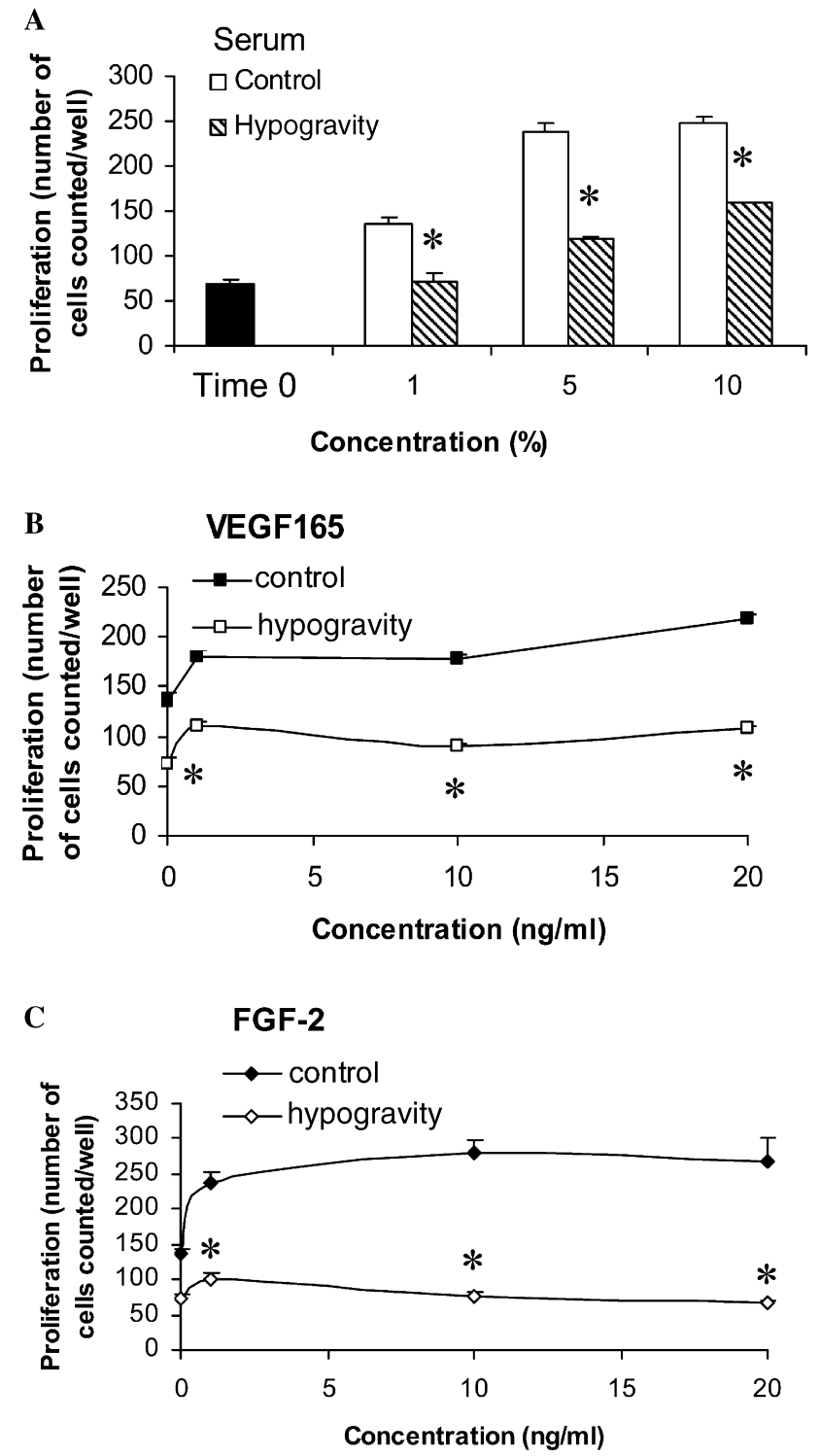

Fig. 4. Effect of hypogravity on endothelial cell proliferation in the presence of serum (A), VEGF (B), and FGF-2 (C). PAEC were exposed to proliferation stimuli after $72 \mathrm{~h}$ incubation in hypogravity and $1 g$ conditions. Increasing concentrations of serum $(1-10 \%)$, VEGF165 (2-20 ng/ml), and FGF-2 (2-20 ng/ml) were added to sparse and synchronized cells. Cell proliferation was measured after $48 \mathrm{~h}$ incubation at $1 \mathrm{~g}$. The number of cells counted at time 0 is reported for reference in (A). Data are expressed as number of cells counted/ well. ${ }^{*} p<0.01$ vs control $1 g$ condition $(n=3)$.

concentrations of VEGF and FGF-2 produced a concentration-dependent increase in cell migration, which was reduced or abrogated in cells previously exposed to simulated hypogravity (Fig. 5). Increasing concentrations of VEGF165 promoted cell migration which was twice as high in control cells relative to hypogravity conditions (Fig. 5A). Similarly, in control cells FGF-2 stimulated cell migration at $50 \mathrm{ng} / \mathrm{ml}$, while it did not in cells previously exposed to hypogravity at any of the concentrations tested (Fig. 5B). 
A

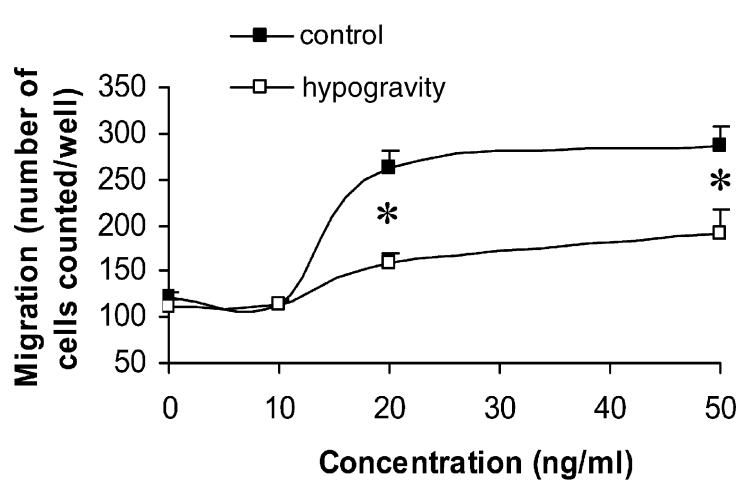

B

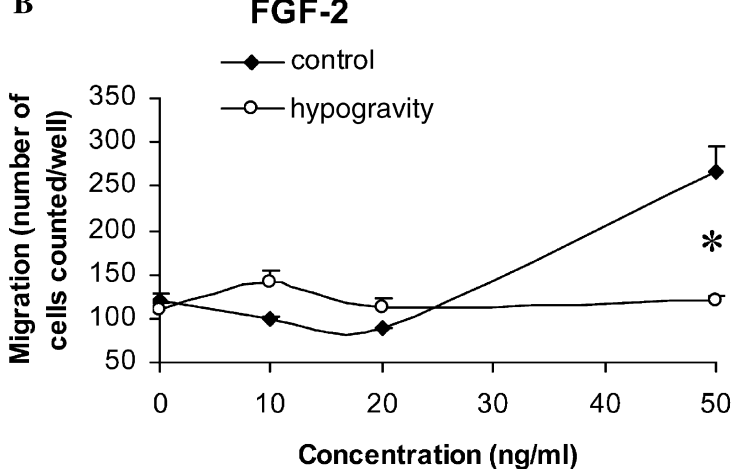

Fig. 5. Effect of hypogravity on endothelial cell migration induced by VEGF (A) and FGF-2 (B). PAEC were challenged for migration after $72 \mathrm{~h}$ incubation in hypogravity and $1 \mathrm{~g}$ conditions. Increasing concentrations of VEGF165 (10-50 ng/ml) and FGF-2 (10-50 ng/ml) were added in each lower well of the microchemotaxis chamber. Cell migration was monitored after $4 \mathrm{~h}$ incubation at $1 g$. Data are expressed as number of migrated cells counted/well. ${ }^{*} p<0.01$ vs control $1 g$ condition $(n=3)$.

From this series of data an irreversible impairment of endothelial cell survival and mobilization was found in cells exposed to simulated hyprogravity.

\section{Discussion}

The results reported in this paper document that hypogravity strongly impairs survival machinery and endothelial cell functions. Following exposure to simulated hypogravity endothelial cells change their morphology and gene expression pattern as well as their functional behaviour, triggering apoptosis signals.

Indeed, the gene profile demonstrates the activation of apoptosis signals triggered by hypogravity as supported by the up-regulation of p53, FAS-L, and BAX genes, and the concomitant down-regulation of the antiapoptotic protein BCL-2 and of the proliferation marker PCNA. We observed an increment of transduction signals (RANK and RANK-L) associated both to osteoclast activation, bone remodelling, and inflammatory cell communications [20]. However, since induction of an angiogenic phenotype has been reported to be associated with the up-regulation of RANK/RANK-L signalling [21,22], conceivably this latter event might be related to a functional recovery of injured cells.

Autofluorescence analysis documents hypogravity-induced morphological alterations, such as change of nucleus shape and dissolution of intracellular organelles, consistent with apoptotic cell death. The disappearance of intracellular organelles, evidenced by the autofluorescence pattern (shown in Fig. 5C), might be attributed to membrane damage, thus explaining the diffuse fluorescence haloes observed in hypogravity cells. It is known that membrane damage causes the leakage of coenzyme molecules, as $\mathrm{NAD}(\mathrm{P}) \mathrm{H}$ and flavins from mitochondria, and their diffusion in all the parts of the cell, nucleus included $[11,23]$. Under $365 \mathrm{~nm}$ excitation, the visible autofluorescence is mainly due to $\mathrm{NAD}(\mathrm{P}) \mathrm{H}$, blue component, and oxidized flavins, yellow-green component [15]. The accumulation of these molecular species highlights a condition of metabolic impairment, due to the dysfunction of mitochondrial electron transport [24]. The increase of $\mathrm{NAD}(\mathrm{P}) \mathrm{Hb}$ by comparing the wavelength ratios $(450 / 480,450 / 525$, and $480 / 525 \mathrm{~nm})$, is consistent with the efflux of the coenzyme from mitochondria, its diffusion and aspecific binding to cytoplasmic and nuclear proteins [23].

The dissolution of mitochondrial membrane integrity with leakage of intra-organelle content into the cytoplasm is related to intrinsic mitochondrial mediated cell death programme [25]. The induction of apoptosis has been reported in different cell lines in simulated hypogravity [26,27]. It is established that cell exposure to both real and simulated hypogravity conditions causes cytoskeleton disorganization associated with microtubule disruption [28]. This results in a failure of mitochondria transport along microtubules, followed by mitochondria clustering and alteration [29]. In accordance with other reports [27], we found an overexpression of Bax. This pro-apoptotic protein is responsible for pore formation in mitochondrial membranes [30]. Permeabilization of the outer mitochondrial membrane causes the release of proapoptotic proteins (i.e., cytochrome $c$ ), able to activate caspases and the apoptotic cascade [31]. Thus, the body of the results obtained in this study fits very well in a frame of apoptosis induced by mitochondrial intrinsic pathway.

Most of the literature data describe a reduction in endothelial cell replication [8], although opposite effects have also been reported [9]. In this study, hypogravity reduced the basal proliferation rate of porcine aortic endothelial cells. Hypogravity conditions caused also a marked impairment of cell responsiveness to exogenous stimuli such as serum or angiogenic factors (VEGF or FGF-2), indicated by their reduced ability to migrate 
and proliferate. This effect was more evident on proliferation than on migration, possibly due to the different duration of the assays ( $4 \mathrm{~h}$ for migration vs $48 \mathrm{~h}$ for proliferation) and experimental conditions (suspended and adherent cells for migration and proliferation, respectively). Moreover, the significant impairment of cell replication exerted by hypogravity may be largely attributed to the disruption of the balance between apoptosis and survival signals, as well as of proliferation and differentiation markers, as evidenced by the gene expression profiling.

The pathophysiological implications of these results are multiple. These findings can explain some of the diseases linked to the weightlessness (as myocardial injury, altered wound healing, osteoporosis, and endocrine organ impairment) and closely associated to endothelial dysfunction [6,7]. The lack of efficacy of angiogenic factors in reverting the basal low proliferation rate of hypogravity exposed cells demonstrates the incapacity of stressed endothelial cells to mount a correct angiogenic response. In this scenario, a reasonable therapeutic strategy for endothelial cell rescue seems the recovery of survival cascades through the enrichment of cells with antiapoptotic proteins (i.e., BH4 domain of $\mathrm{Bcl}-2$ ), as recently proposed in oxidative stress and ischemic conditions $[25,32]$.

In summary, the experiments reported here document the cellular and molecular mechanisms of endothelial cell alterations in low gravity and may provide useful tools to design and validate novel therapeutic strategies for weightlessness related diseases.

\section{Acknowledgments}

The work was partially funded by the University of Siena (PAR funding) to L.M. and M.Z. and MIUR FIRB project $n$. RBNE01458S_007 to M.Z. The technical support of Dr. S. Pezzatini (Univ. Siena), G. Romano and V. Basile (Univ. Florence), and Prof. P.A. Bernabei (Careggi Hospital, Florence), and the helpful discussion of Prof. A. Giachetti (Lifetech Srl, Florence) are also greatly acknowledged.

\section{References}

[1] P. Carmeliet, Mechanisms of angiogenesis and arteriogenesis, Nat. Med. 6 (2000) 389-395.

[2] P. Carmeliet, R.K. Jain, Angiogenesis in cancer and other diseases, Nature 407 (2000) 249-257.

[3] N. Ferrara, H.P. Gerber, J. LeCouter, The biology of VEGF and its receptors, Nat. Med. 9 (2003) 669-676.

[4] I.S. Syed, T.A. Sanborn, T.K. Rosengart, Therapeutic angiogenesis: a biologic bypass, Cardiology 101 (2004) 131-143.

[5] M.A. Gimbrone Jr., J.N. Topper, T. Nagel, K.R. Anderson, G. Garcia-Cardena, Endothelial dysfunction, hemodynamic forces, and atherogenesis, Ann. N.Y. Acad. Sci. 902 (2000) 230-239.
[6] M.E. Kirchen, K.M. O’Connor, H.E. Gruber, J.R. Sweeney, I.A. Fras, S.J. Stover, A. Sarmiento, G.J. Marshall, Effects of microgravity on bone healing in a rat fibular osteotomy model, Clin. Orthop. 318 (1995) 231-242.

[7] M.K. Henry, B.R. Unsworth, V. Sychev, T.S. Guryeva, O.A. Dadasheva, S.J. Piert, K.E. Lagel, L.C. Dubrovin, G.C. Jahns, K. Boda, V. Sabo, M.M. Samet, P.I. Lelkes, Launch conditions might affect the formation of blood vessels in the quail chorioallantoic membrane, Folia Vet. 42 (1998) S25-S31.

[8] L.B. Buravkova, Y.A. Romanov, The role of cytoskeleton in cell changes under condition of simulated microgravity, Acta Astronaut. 48 (2001) 647-650.

[9] S.I. Carlsson, M.T. Bertilaccio, E. Ballabio, J.A. Maier, Endothelial stress by gravitational unloading: effects on cell growth and cytoskeletal organization, Biochem. Biophys. Acta 1642 (2003) 173-179.

[10] M. Dellinger, M. Geze, R. Santus, E. Kohen, C. Kohen, J.G. Hirschberg, M. Monti, Imaging of cells by autofluorescence: a new tool in the probing of biopharmaceutical effects at the intracellular level, Biotechnol. Appl. Biochem. 28 (1998) 25-32.

[11] P.X. Petit, M.-C. Gendron, N. Schrantz, D. Métivier, G. Kroemer, Z. Maciorowska, F. Sureau, S. Koester, Oxidation of pyridine nucleotides during Fas- and ceramide-induced apoptosis in Jurkat cells: correlation with changes in mitochondria, glutathione depletion, intracellular acidification and caspase 3 activation, Biochem. J. 353 (2001) 357-367.

[12] J. Kroll, J. Waltenberger, VEGF-A induces expression of eNOS and iNOS in endothelial cells via VEGF receptor-2 (KDR), Biochem. Biophys. Res. Commun. 252 (1998) 743-746.

[13] T. Hoson, S. Kamisaka, Y. Masuda, M. Yamashita, B. Buchen, Evaluation of the three-dimensional clinostat as a simulator of weightlessness, Planta 203 (1997) S187-S187.

[14] K.J. Livak, D.T. Schmittgen, Analysis of relative gene expression data using real time quantitative PCR and the $2^{-\Delta \Delta C_{\mathrm{t}}}$ method, Methods 25 (2001) 402-408.

[15] R. Richards-Kortum, E. Sevick-Muraca, Quantitative optical spectroscopy for tissue diagnosis, Ann. Rev. Phys. Chem. 47 (1996) 555-606.

[16] F. Fusi, G. Agati, M. Monici, R. Pratesi, S. Romano, P.A. Bernabei, Multicolor imaging autofluorescence microscopy: a new technique for the discrimination of normal and neoplastic tissues and cells, Recent Res. Dev. Photochem. Photobiol. 6 (2002) 7993.

[17] L. Morbidelli, S. Donnini, F. Chillemi, A. Giachetti, M. Ziche, Angiosuppressive and angiostimulatory effects exerted by synthetic partial sequences of endostatin, Clin. Cancer Res. 9 (2003) 5358-5369.

[18] R. Friesel, T. Maciag, Fibroblast growth factor prototype release and fibroblast growth factor receptor signaling, Thromb. Haemost. 82 (1999) 748-754.

[19] L. Morbidelli, R. Birkenhaeger, W. Roeckl, H.J. Granger, U. Kaerst, H. Weich, M. Ziche, Distinct capillary density and progression promoted by vascular endothelial growth factor-A homodimers and heterodimers, Angiogenesis 1 (1997) $117-130$.

[20] L.E. Theill, W.J. Boyle, J.M. Penninger, RANK-L and RANK: T cells, bone loss, and mammalian evolution, Ann. Rev. Immunol. 20 (2002) 795-823.

[21] J.K. Min, Y.M. Kim, Y.M. Kim, E.C. Kim, Y.S. Gho, I.J. Kang, S.Y. Lee, Y.Y. Kong, Y.G. Kwon, Vascular endothelial growth factor up-regulates expression of receptor activator of NF-kappa B (RANK) in endothelial cells. Concomitant increase of angiogenic responses to RANK ligand, J. Biol. Chem. 278 (2003) 39548-39557.

[22] H.H. Kim, H.S. Shin, H.J. Kwak, K.Y. Ahn, J.H. Kim, H.J. Lee, M.S. Lee, Z.H. Lee, G.Y. Koh, RANKL regulates endothelial cell survival through the phosphatidylinositol $3^{\prime}$-kinase/Akt signal transduction pathway, FASEB J. 17 (2003) 2163-2165. 
[23] K. Konig, T. Krasieva, E. Bauer, U. Fiedler, M.W. Berns, B.J. Tromberg, K.O. Greulich, Cell damage by UVA radiation of a mercury microscopy lamp probed by autofluorescence modifications, cloning assay and comet assay, J. Biochem. Opt. 1 (1996) 217 222.

[24] R.M. Duchen, T.J. Biscoe, Mitochondrial function in type I cells isolated from rabbit arterial chemoreceptors, J. Physiol. 450 (1992) 13-31.

[25] S. Shimizu, A. Konishi, T. Kodama, Y. Tsujimoto, BH4 domain of anti-apoptotic Bcl-2 family members closes voltage-dependent anion channel and inhibits apoptotic mitochondrial changes and cell death, Proc. Natl. Acad. Sci. USA 97 (2000) 3100-3105.

[26] D. Grimm, J. Bauer, P. Kossmehl, M. Shakibaei, J. Schoberger, H. Pickenhahn, G. Schulze-Tanzil, R. Vetter, C. Eilles, M. Paul, A. Cogoli, Simulated microgravity alters differentiation and increases apoptosis in human follicular thyroid carcinoma cells, FASEB J. 16 (2002) 604-606.

[27] P. Kossmehl, M. Shakibaei, A. Cogoli, M. Infanger, F. Curcio, J. Schonberger, C. Eilles, J. Bauer, H. Pickenhahn, G. SchulzeTanzil, M. Paul, D. Grimm, Weightlessness induced apoptosis in normal thyroid cells and papillary thyroid carcinoma cells via extrinsic and intrinsic pathways, Endocrinology 144 (2003) 4172 4179.

[28] C. Papaseit, N. Pochon, J. Tabony, Microtubule self-organization is gravity-dependent, Proc. Natl. Acad. Sci. USA 97 (2000) 8364 8368 .

[29] H. Schatten, M.L. Lewis, A. Chakrabarti, Spaceflight and clinorotation cause cytoskeleton and mitochondria changes and increases in apoptosis in cultured cells, Acta Astronaut. 49 (2001) 399-418.

[30] A. Gross, J.M. McDonnell, S.J. Korsmeyer, Bcl-2 family members and the mitochondria in apoptosis, Genes Dev. 13 (1999) 18991911.

[31] C. Fumarola, G.G. Guidotti, Stress-induced apoptosis: toward a symmetry with receptor-mediated cell death, Apoptosis 9 (2004) $77-82$.

[32] S. Cantara, S. Donnini, A. Giachetti, P.E. Thorpe, M. Ziche, Exogenous $\mathrm{BH} 4 / \mathrm{Bcl}-2$ peptide reverts coronary endothelial cell apoptosis induced by oxidative stress, J. Vasc. Res. 41 (2004) 202 207.

[33] G. Wyszecki, W.S. Stiles, Color Science: Concepts and Methods, Quantitative Data and Formulae, second ed., Wiley, New York, 1982. 